\title{
Sistema para monitoramento de potência aparente alternativo conectado à internet
}

\author{
Maycon Jean de Moura ${ }^{[1]}$ e Francisco Magno Monteiro Sobrinho ${ }^{[2]}$ \\ [1] Universidade Federal Rural do Semi-árido; mayconjeanm@gmail.com \\ ${ }^{[2]}$ Universidade Federal Rural do Semi-árido; magno.monteiro@ufersa.edu.br \\ Recebido: 17/06/2019; \\ Aceito: 13/07/2019; \\ Publicado: 07/10/2019.
}

Resumo: Com as crises hídricas vividas nos últimos anos e o consequente aumento nas tarifas de consumo de energia elétrica brasileira, impacta a crescente busca por eficiência energética. O presente trabalho tem como objetivo desenvolver um dispositivo de baixo custo para monitorar a potência aparente de residências monofásicas de baixa tensão possibilitando o acompanhamento dos valores medidos em tempo real remotamente pela internet e da visualização com a resolução de segundos para que seja possível tomar conhecimento, indiretamente, do consumo diário, algo quase impossível atualmente já que a concessionária fornece os dados mensalmente e é dispendioso coletar os dados manualmente. Foi alcançado o objetivo proposto, verificando o reduzido custo do projeto, a robustez do servidor e do software usado para processar e exibir os dados

Palavras-chave: NodeMCU; Monitoramento remoto; Medidor de potência aparente

\section{INTRODUÇÃO}

$\mathrm{E}_{\mathrm{d}}^{\mathrm{n}}$ m menos de dez anos, a energia elétrica aumentou em $10 \%$ a sua participação no consumo residencial final de energia e em $5 \%$ no consumo energético total do setor comercial [1]. Um crescimento constante mesmo com variações negativas do PIB no período. Além disso, as crises hídricas, que afetam a principal fonte usada na geração de energia elétrica brasileira, aumenta o valor pago pelo consumo, tornando interessante o seu uso eficiente da mesma até mesmo para pequenos consumidores. Dado esta crescente busca por eficiência, o setor pode gerar negócios de US\$ 260 bilhões e mais de 1,2 milhão de empregos direta e indiretamente, o que mostra a preocupação da sociedade com o tema [2]. Com isso em mente, existe alguma forma de aumentar a eficiência energética de uma residência a um baixo custo?

Uma das formas de aumentar a eficiência energética seria evitar desperdícios e, para isso, é necessário conhecer bem o consumo a todo momento para que gastos desnecessários sejam evitados e se crie consciência sobre o uso da energia elétrica. Além disso, com a criação de novas tarifas como a tarifa branca, que criar tarifas diferentes para períodos do dia e dias diferentes [3], torna-se imprescindível a visualização do consumo em uma resolução maior. A concessionária fornece, através de sua fatura, o valor consumido nos últimos 12 meses, o que não é suficiente para identificar problemas pontuais e nem grandes problemas forma rápida, já que o reflexo será visto apenas na fatura do mês posterior. Com isso, o consumidor que em sua maioria considera o preço da energia muito caro ou caro não consegue visualizar formas de reduzir o consumo [4].

Então, para solucionar esse problema, foi proposto um sistema no qual será ligado à entrada de energia elétrica para monitorar a potência aparente consumida e enviar esses dados para a nuvem, para que os mesmos sejam exibidos em gráficos de fácil visualização para que o usuário possa identificar possíveis perdas e problemas no circuito em um tempo menor do que seria possível com os dados da concessionária, podendo haver o aumento do aproveitamento de energia elétrica em questão. Tal ideia pode ser encaixada em uma das definições possível de para o Smart-meter [5], um dispositivo que vai além do medidor convencional, já que seria possível observar o consumo em tempo real e de qualquer lugar. 
O presente trabalho tem como objetivo desenvolver um dispositivo de baixo custo para o monitoramento da potência aparente consumida em uma residência monofásica de $220 \mathrm{~V}$ com uma resolução de apenas poucos segundos, maior que a fornecida pela concessionária. Também será criado um aplicativo para exibir os dados medidos de fácil visualização e interpretação por meio de gráficos para resumir a grande quantidade de dados obtidos com a medição.

\section{MATERIAIS E MÉTODOS}

O projeto foi dividido em dois módulos, como é possível observar na Figura 2, para facilitar o entendimento e separar partes fundamentais do mesmo. Ele consiste em um medidor de corrente e uma forma de enviar os dados para o servidor e processá-los. Não é possível obter a potência apenas com os dados enviados e para isso, assumiuse que a tensão na rede seria $220 \mathrm{~V}$. Essa consideração foi feita pois a placa usada tem apenas uma entrada com ADC, que será usada para medir a corrente eficaz do circuito. Com essa consideração é possível usar apenas a placa NodeMCU para a prototipagem, reduzindo custos e simplificando o modelo, mas sem perder a qualidade da informação.

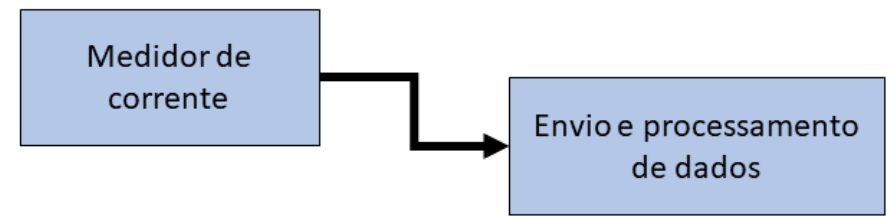

FIGURA 2. Esquema proposto para o trabalho. (Autoria própria)

\subsection{Medidor de corrente}

Para a medição da corrente foi utilizado o SCT-013, um transformador de corrente que suporta até 100A com saída máxima de 50mA. Como é possível observar, a tensão induzida será alternada, já que a forma da corrente é senoidal. Com isso é necessário realizar adaptações para que o ADC, que tem tensão máxima de 3,3V e mínima de $0 \mathrm{~V}$, consiga medir com a melhor resolução possível sem ultrapassar os parâmetros máximos do mesmo. Para isso, além do transformador de corrente é necessário um circuito para aquisição dos dados do sensor de corrente, como pode ser visto na Figura 3.

Como a saída do SCT-013 é uma corrente que depende da corrente de entrada é preciso utilizar um resistor shunt para que possa ser medido uma tensão correspondente a corrente na saída do transformador que corresponde, respectivamente a uma corrente no condutor medido.

Assim, como o seno tem uma simetria ímpar é preciso elevar a tensão para que o sinal se torne contínuo. Assim, para obter a maior resolução possível foi escolhido elevar a mesma para metade da tensão máxima para que a distância entre o centro do sinal seja máxima. Foi usado dois resistores iguais como divisor de tensão conectados em 3,3V para obter a componente DC do sinal. Já o resistor shunt foi projetado para que a corrente de pico máxima, somada com a componente DC, não ultrapasse a tensão máxima suportada pelo ADC. Assim,

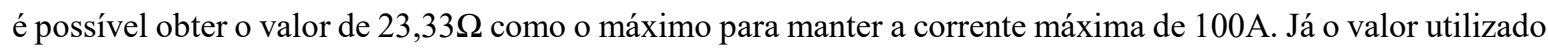
foi menor do que o calculado, proporcionando uma menor resolução, mas garantindo que mesmo na corrente máxima. a entrada ADC não receberá uma tensão maior que a tensão máxima. Como o resistor utilizado foi de $22 \Omega$, a corrente máxima eficaz medida pelo ESP será de 106A, podendo assim usar toda a capacidade do sensor. $\mathrm{O}$ circuito final pode ser visto na Figura 4 onde A0 é o pino analógico do NodeMCU. 


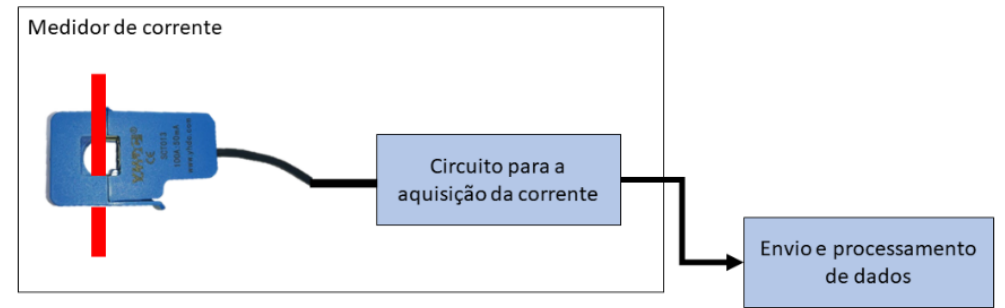

FIGURA 3. Esquema resumido do módulo medidor de corrente. (Autoria própria)

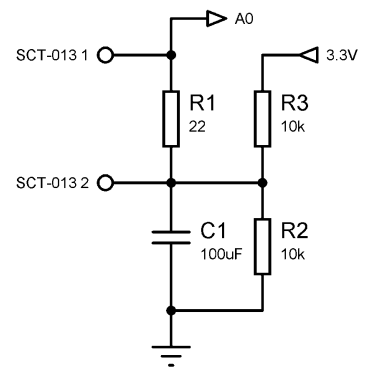

FIGURA 4. Circuito para a aquisição da corrente. (Autoria própria)

Para o cálculo da corrente eficaz foi utilizado a biblioteca Emonlib, que é aberta e para Arduino, de um projeto de monitor de energia de código aberto. Assim é possível abstrair o cálculo computacional realizado pela biblioteca reduzindo a complexidade do projeto.

\subsection{Envio e processamento de dados}

Já para o meio do envio dos dados, foi escolhido o Wifi, utilizando a principal vantagem do ESP que é já contar com um módulo Wifi integrado. E para armazenar os dados foi escolhido o Realtime database do Firebase, que por sua simplicidade e atualizações em tempo real se encaixou perfeitamente nos requisitos do projeto, sendo mais eficientes que banco de dados relacionais tradicionais nesse tipo de situação [19]. Os dados escolhidos para serem enviados foram: data, corrente. Com eles é possível calcular a potência aparente, plotar gráficos em relação ao tempo em qualquer intervalo de tempo disponível e identificar cada momento do dia no gráfico de potência aparente instantânea. A Figura 5 mostra um resumo do processo de envio e processamento de dados.

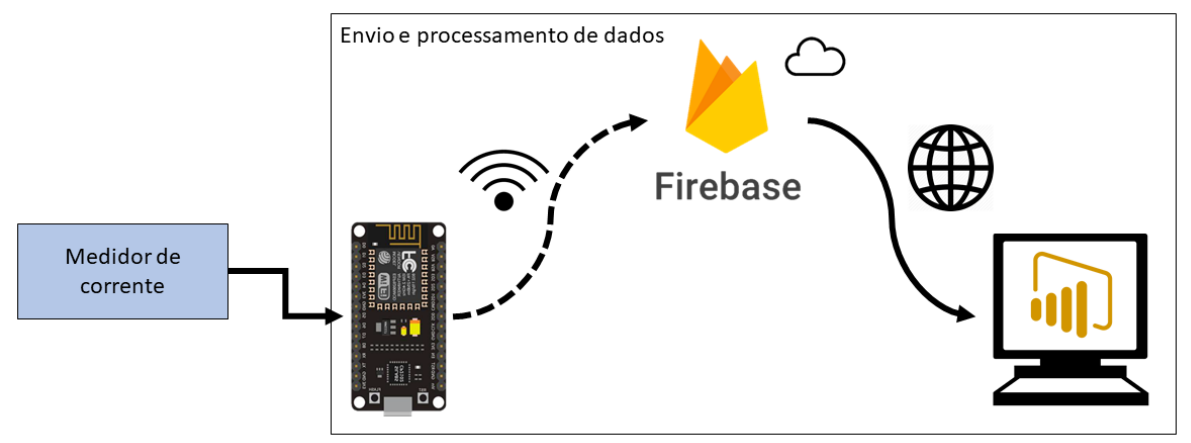

FIGURA 5. Esquema resumido do processo de envio e processamento de dados. (Autoria própria)

Como a data da medição é enviada, é possível ter um atraso no envio sem que o dado perca seu valor, já que não depende da hora que entra no servidor. A data é obtida em Unix Timestamp de um servidor ntp, que significa 
Network Time Protocol ou Protocolo de Tempo para Redes e permite a sincronização de dispositivos eletrônicos em uma rede [20], sendo utilizado um servidor brasileiro para reduzir a latência. Com isso é possível enviar toda a informação de data e hora em apenas um inteiro de fácil interpretação para o computador.

O dispositivo foi configurado para medir a corrente eficaz no circuito em intervalor e 10s para que fosse possível executar todo o programa nele presente e para que o servidor não ficasse sobrecarregado de informações já que em apenas um dia seriam enviadas mais de 8000 requisições com objetos contendo os dados mencionados.

Como o Firebase dispõe de uma api RESTful para acessar os dados armazenados, foi escolhido o Power BI como visualizador. Assim, para o envio seria feito uma requisição http do tipo POST onde é passado como parâmetro o objeto Json contendo a data e o valor da corrente medida pelo circuito. Já para a leitura, feita pelo Power BI, é realizada uma requisição GET que retorna todos os elementos salvos no banco em Json, essa resposta é reordenada em tabelas e seus dados, valor da corrente e da data, convertidos em valores de fácil leitura para humanos. Assim, é possível usar os dados tratados no Power BI para criação de representações que sintetizem as informações enviadas pelo ESP para o servidor de forma clara e simples. Podendo assim ser usada para a conscientização e redução do consumo por parte dos usuários.

\subsection{Aquisição de dados}

Para testar o protótipo, o mesmo foi ligado a um quadro geral de distribuição, como mostra a Figura 6, durante o período de 28 de outubro a 22 de dezembro. O teste se propunha observar a resposta do servidor e dos dispositivos ao uso contínuo durante o período do mesmo. Assim, os dados enviados para o servidor foram a corrente medida e a data da medição em Unix Timestamp. Além disso, foi também colhido o uso do servidor durante o período para que fosse possível entender o impacto do constante envio de dados para o mesmo. Para a alimentação e para reduzir a quantidade de componentes que podiam ocasionar erro, foi utilizado uma fonte comercial com saída MicroUSB para alimentar o NodeMCU durante o teste.

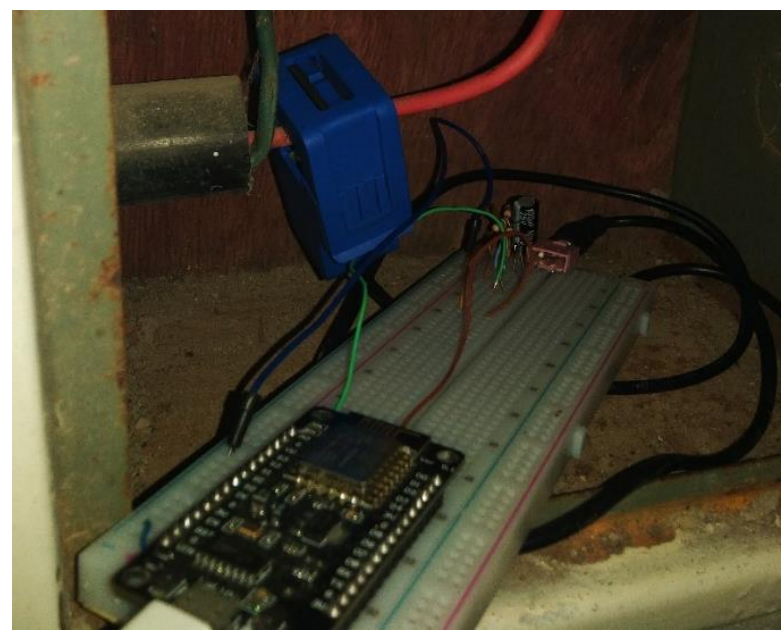

FIGURA 6. Protótipo montado no quadro de distribuição. (Autoria própria) 


\section{RESUltAdOs E DisCUSSÕES}

\subsection{Custo total do circuito}

A Tabela 1 resume os valores unitários de cada componente e o total gasto na confecção do protótipo do circuito projetado. Foi priorizado componentes de baixo custo e de acesso local para a fácil construção do dispositivo.

TABELA 1. Custo de componentes utilizados na construção do protótipo. (Autoria própria)

\begin{tabular}{ccc}
\hline Quantidade & Componente & Custo Unitário \\
\hline 1 & NodeMCU & $\mathrm{R} \$ 49,90$ \\
\hline 1 & SCT-013 & $\mathrm{R} \$ 59,90$ \\
\hline 3 & Resistores de $1 / 4 \mathrm{~W}$ & $\mathrm{R} \$ 0,25$ \\
\hline 1 & Conector P2 & $\mathrm{R} \$ 2,00$ \\
\hline 1 & Capacitor cerâmico & $\mathrm{R} \$ 0,40$ \\
\hline & Total & $\mathrm{R} \$ 112,95$
\end{tabular}

O custo final do protótipo poderia ser reduzido consideravelmente se os componentes fossem adquiridos em sites de compras internacionais. Mas mesmo com os componentes comprados, o valor do protótipo é uma fração da alternativa internacional e representa menos do que quatro meses de mensalidade do Ecomonitor. Os valores mostrados na Tabela 1 são de componentes acabados e próprios para a prototipagem, o custo real de um dispositivo com as mesmas características seria menor já que não seriam necessárias todas as interfaces por eles oferecidas, podendo, assim, usar dispositivos mais baratos como o próprio ESP8266 no lugar do NodeMCU, com a devida adaptação, mas sem perder qualidade no produto final.

\subsection{Processamento de dados}

Para processar e analisar os dados provenientes do medidor, foi criado uma página no Power BI, para resumir os dados medidos. Foi usado dados medidos entre outubro e dezembro para a validação da página. A Figura 7 , 8 e 9 mostra alguns gráficos gerados com os dados medidos durante o teste do módulo medidor de corrente e do envio e processamento de dados, o que corresponde a um total de 475 mil objetos com data e valor da corrente. Com os referidos dados é possível criar várias visualizações, inclusive por períodos menores ou com resoluções diferentes e períodos posteriormente determinados, como a Figura 9, que apresenta um agrupamento de intervalos de horas para representar a manhã, tarde, noite e madrugada.

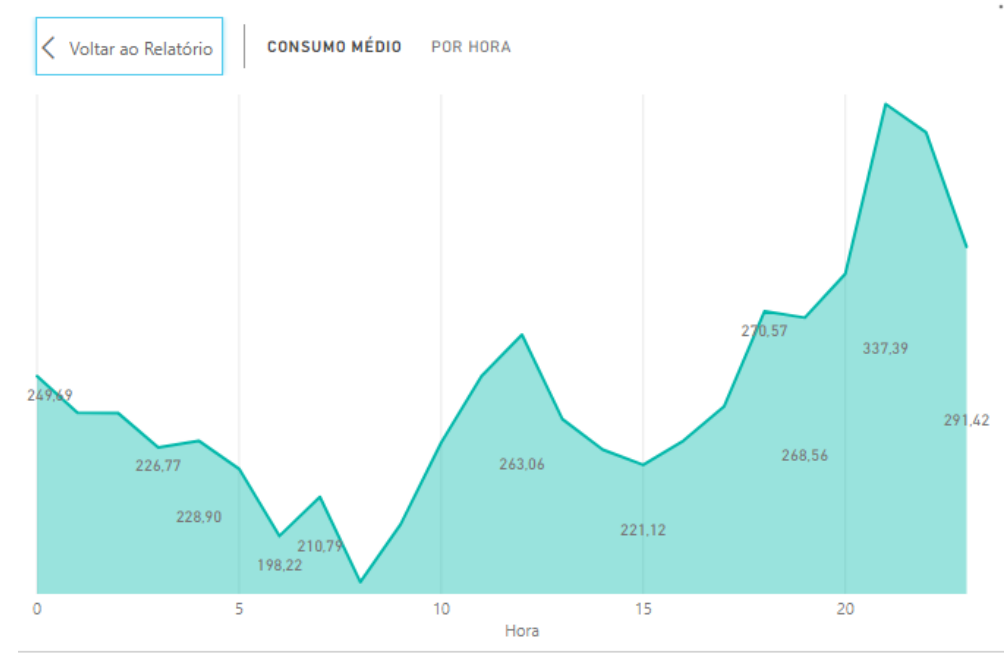

FIGURA 7. Gráfico de linha da potência aparente média do período por hora. (Autoria própria) 


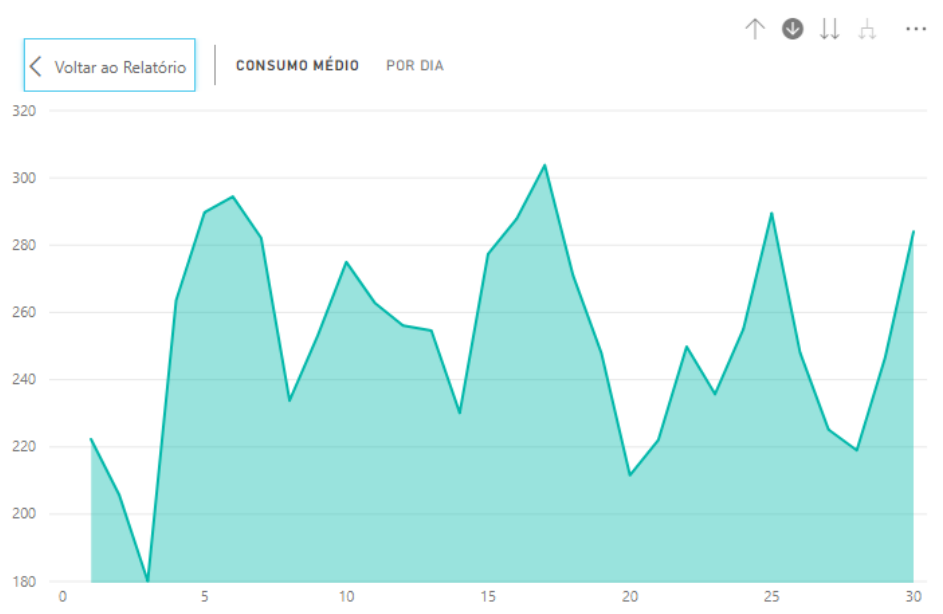

FIGURA 8. Gráfico de linha da potência média de novembro por dia. (Autoria própria)

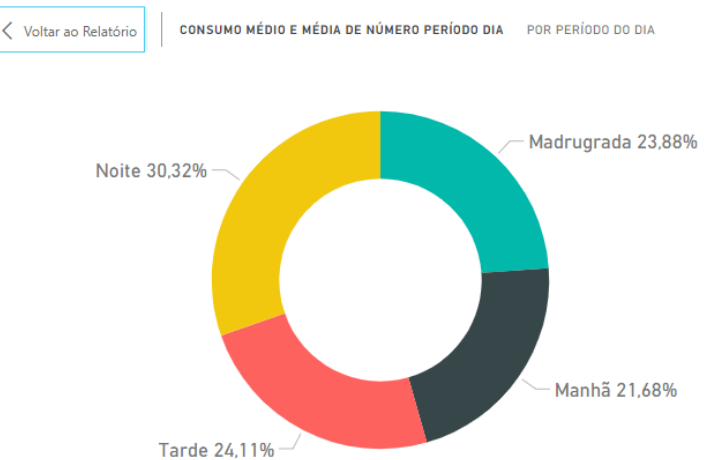

FIGURA 9. Contribuição da potência aparente em cada momento do dia. (Autoria própria)

Além disso, é possível perceber pela Figura 10 que mesmo com o volume gigantesco de dados enviados, o armazenamento do banco de dados quase não foi utilizado, devido a forma que o dado foi passado. Mas houve um grande volume de downloads para os dados apresentados, isso se deve ao fato do Power BI, ao buscar novamente os dados para atualizar seus gráficos, baixa todos os dados novamente, provocando um alto tráfego de downloads proporcional a quantidade requisições e de dados disponíveis.

Armazenamento (2)

$27,7 \mathrm{MB}$
Downloads (2)

$83,4 \mathrm{MB}$ Total de 30 dias
Carregar (?)

$16 \%$ pico

FIGURA 10. Valores medidos no banco de dados. (Autoria própria)

\section{CONCLUSÃO}

O presente trabalho se propôs a projetar e construir um medidor de potência aparente com um baixo custo para uma residência de baixa tensão. Obtendo êxito em seus objetivos propostos, os dados apresentados refletem, em parte, o consumo do local já que o valor medido foi a potência aparente. O circuito proposto tem um custo muito baixo, tendo o seu custo de material próximo ao valor de quatro mensalidades pagas pelo aluguel de equipamentos similares disponíveis no mercado nacional ou de uma fração da alternativa internacional. $\mathrm{O}$ Power BI é uma ótima ferramenta para analisar os dados, mas não foi possível utilizar a função de tempo real, 
já que a atualização automática nos dados só está disponível na versão paga do software. O que resolveria o problema do grande volume de download no banco, mesmo que esse problema possa ser ignorado em pequenas escalas. Mesmo assim, o programa apresentou um comportamento excelente trabalhando com quase meio milhão de entradas.

Como sugestão para futuros trabalhos fica o estudo de formas para medir a potência ativa de forma barata para, assim, medir o consumo real da residência monofásica.

\section{REFERÊNCIAS}

[1] EPE - Empresa de Pesquisa Energética. Balanço Energético Nacional 2018 - Ano base 2017. Rio de Janeiro, 2018. Disponível em: <http://www.epe.gov.br/pt> Acesso em 7 de novembro de 2018.

[2] ASSOCIAÇÃO BRASILEIRA DAS EMPRESAS DE SERVIÇOS DE CONSERVAÇÃO DE ENERGIA (São Paulo). Abesco - Eficiência Energética| Associação Brasileira das Empresas de Serviços de Conservação de Energia, com foco em Eficiência Energética. 2018. Disponível em: <http://www.abesco.com.br/>. Acesso em: 5 nov. 2018. Autor 1, A.; Autor 2, B. Título do Livro, 3rd ed.; Editora: Editora Local, País, Ano; p. 00-00.

[3] ASSESSORIA DE IMPRENSA. Agência Nacional de Energia Elétrica. Tarifa branca é nova opção para quem tem consumo acima de $250 \mathrm{KWh}$. Disponível em: <http://www.aneel.gov.br/sala-de-imprensaexibicao-2/-/asset_publisher/zXQREz8EVlZ6/content/tarifa-branca-e-nova-opcao-para-quem-temconsumo-acima-de-250-kwh/656877?inheritRedirect=false>. Acesso em: 11 fev. 2019

[4] IBOPE INTELIGÊNCIA. Pesquisa de Opinião Pública: Energia Elétrica. Abril de 2018. Disponível em: <http://www.queroenergialivre.com.br/wp-content/uploads/2018/08/Ibope-Abraceel-2018.pdf>

Acesso em: 8 de novembro de 2018.

[5] KOPONEN, Pekka et al. Definition of Smart Metering and Applications and Identification of Benefits. 1.1 Finland: European Smart Metering Alliance Authors, 2008.

[6] CESÁRIO JÚNIOR, José Maria. UM MEDIDOR DE ENERGIA ELÉTRICA INTEGRADO EM REDES DE COMUNICAÇÕES Dissertação. 2014. 95 f. Dissertação (Mestrado) - Curso de Mestrado em Tecnologia, Universidade Estadual de Campinas, Limeira, 2014.

[7] OPENENERGYMONITOR. EmonPi. Disponível em: <https://openenergymonitor.com/emonpi-3/>. Acesso em: 11 fev. 2019.

[8] ECOMONITOR (Rio Grande do Sul). Ecomonitor: Monitoramento do consumo de energia e água, a qualquer momento, em qualquer lugar!. Disponível em: <http://ecomonitor.com.br〉. Acesso em: 12 fev. 2019.

[9] SYSTEMS, Espressif. ESP8266EX: Datasheet. 6. ed. Espressif Systems. 2018.

[10] ALIEXPRESS. ESP8266 serial WIFI model ESP-12E Authenticity Guaranteed Upgraded version.

[11] SQUIX78. ESP8266 module comparison: ESP-01, ESP-05, ESP-12, ESP-201, Test Board and NodeMCU. Disponível em: <https://blog.squix.org/2015/03/esp8266-module-comparison-esp-01-esp-05.html>. Acesso em: 28 jan. 2019.

[12] FEZARI, Mohamed. NodeMCU V3 For Fast IoT Application Development. Outubro, 2018.

[13] KOON, W. "Current Sensing for Energy Metering". Analog Devices. Disponível em http://www.analog.com/static/imported-

files/recommended_reading/688920132Current_sensing_for_meteringNew.pdf. Último acesso em $11 / 12 / 2013$

[14] REDDY, M Sai Prathap. Implementing smart home using firebase. v. 6, n. 10, p. 193-199, 2016.

[15] FIREBASE. Firebase Realtime Database. Disponível em: <https://firebase.google.com/docs/database/?hl=pt-br>. Acesso em: 10 fev. 2019.

[16] JSON.ORG. Introducing JSON. Disponível em: <https://www.json.org>. Acesso em: 09 fev. 2019.

[17] LI, Yishan; MANOHARAN, Sathiamoorthy. A performance comparison of SQL and NoSQL databases. 2013 Ieee Pacific Rim Conference On Communications, Computers And Signal Processing (pacrim), [s.1.], p.14-19, ago. 2013.

[18] COLABORADORES. O que é Power BI? Disponível em: <https://docs.microsoft.com/pt-br/powerbi/power-bi-overview>. Acesso em: 09 fev. 2019.

[19] VEEN, Jan Sipke Van Der; WAAIJ, Bram Van Der; MEIJER, Robert J. Sensor Data Storage Performance : SQL or NoSQL, Physical or Virtual. 2012 IEEE Fifth International Conference on Cloud Computing, p. 431-438, 2012. 
[20] NTP.BR. O NTP. Disponível em: <https://ntp.br/ntp.php>. Acesso em: 09 fev. 2019. 